\title{
Qualidade de vida e qualidade de sono na insuficiência cardíaca
}

\author{
Quality of life and quality of sleep in heart failure
}

\author{
Layana Marques de Oliveirar, ${ }^{1,2}$ Tamara Martins da Cunhal; Ivan Daniel Bezerra Nogueira ${ }^{1,2}$; \\ Patrícia Angélica de Miranda Silva Nogueira ${ }^{1,2}$ \\ ${ }^{1}$ Departamento de Fisioterapia, Universidade Federal do Rio Grande do Norte - UFRN. Natal, RN -Brasil. \\ ${ }_{2}^{2}$ Programa de Pós-graduação de Fisioterapia - Universidade Federal do Rio Grande do Norte -UFRN. Natal, RN - Brasil. \\ Endereço para Correspondência: \\ Patrícia Angélica de Miranda Silva Nogueira, \\ Av. Senador Salgado Filho, 3000. Caixa Postal 1524 \\ 59072-970 - Natal - RN [Brasil] \\ idpa02@ufrnet.br/idpa01@hotmail.com
}

\begin{abstract}
Resumo
Introdução: A insuficiência cardíaca (IC) é uma síndrome clínica complexa, caracterizada pela incapacidade do ventrículo esquerdo bombear sangue suficiente para o corpo. Dentre as características clínicas da IC estão a baixa qualidade de vida e de sono. Objetivo: Relacionar a qualidade de vida $(\mathrm{QV})$ e a qualidade de sono (QS) com capacidade funcional de pacientes com IC e saudáveis. Métodos: Estudo transversal observacional, no qual foram avaliados 21 pacientes com IC (grupo IC) e 21 indivíduos auto relatados saudáveis (grupo controle). Foram avaliadas a QV e QS, a sonolência diurna excessiva (SDE) e a distância percorrida do teste da caminhada de 6 minutos (TC6M). Resultados: Foi observado uma melhor QV e QS no grupo IC em comparação ao grupo controle $(\mathrm{p}<0,05)$. Encontramos correlações positivas entre o capacidade funcional e limitação por aspectos emocionais com a distância percorrida no TC6M ( $\mathrm{r}=0,475, \mathrm{p}=0,034)$ e $(\mathrm{r}=0,620$, $\mathrm{p}=0,004$,$) , respectivamente. Conclusão: Nossos resultados demonstram que a IC$ gera impactos negativos na QV e na QS da amostra estudada.
\end{abstract}

Descritores: Insuficiência Cardíaca; Qualidade de Vida; Sono.

\begin{abstract}
Introduction: Heart failure (HF) is a complex clinical syndrome characterized by the inability of the the left ventricle to pump enough blood into the body. Among the clinical characteristics of HF are the low quality of life and quality of sleep. Objective: To relate quality of life (QOL) and sleep quality (SQ) with functional capacity of patients with HF and healthy individuals. METHODS: A cross-sectional observational study in which 21 patients with HF (group HF) and 21 self-reported healthy individuals were evaluated. The QOL and SQ, excessive daytime sleepiness (EDS) and the 6-minute walk test (6MWT) were measured. Results: A better QOL and SQ were observed in the HF group compared to the control group $(\mathrm{p}<0.05)$. We found positive correlations between FC and AE with distance walked on 6MWT $(r=0.475, \mathrm{p}=0.034)$ and $(\mathrm{r}=0.620, \mathrm{p}=0.004)$, respectively. Conclusion: Our results demonstrate that the HF generates negative impacts on the QOL and SQ of the sample studied.
\end{abstract}

Keywords: Heart Failure; Quality of life; Sleep. 


\section{Introdução}

A insuficiência cardíaca (IC) é uma síndrome clínica complexa que pode resultar de qualquer doença cardíaca estrutural ou funcional, prejudicando a capacidade do ventrículo para encher ou ejetar o sangue ${ }^{1}$. Essa síndrome é caracterizada pela baixa tolerância ao exercício, na maioria das vezes, associada à fadiga e à dispneia $^{2,3}$. Como resultado de tais fatores, há um aumento progressivo da inatividade física, causando descondicionamento físico, o qual contribui para elevar os sintomas e a intolerância ao exercício ${ }^{4}$. A limitação da funcionalidade comumente acarreta incapacidades funcionais, redução na qualidade de vida (QV), e um inevitável aumento da mortalidade nessa população ${ }^{5,6}$. Em adição as consequências da limitação funcional, estudos mostram uma alta prevalência de distúrbios do sono em pacientes com IC. Tais distúrbios foram considerados indicadores da severidade da falência cardíaca nos pacientes com IC da população europeia ${ }^{7-10}$. As apneias e despertares periódicos fragmentam o sono fortemente, exacerbando a fadiga e causando sonolência diurna excessiva (SDE). A SDE é conceituada como a dificuldade em manter um nível satisfatório de vigília com uma sensação de estar sonolento de forma inapropriada, podendo interferir nas atividades profissionais, familiares e em relacionamentos sociais ${ }^{11}$.

$\mathrm{Na} I C$, a qualidade de sono (QS) está relacionada com a capacidade funcional dos pacientes. Os sintomas primários da IC, como fadiga e dispneia, interferem diretamente na QS causando SDE, que comprometem ainda mais a capacidade funcional, que já é prejudicada pela patologia ${ }^{12}$.

Nesse contexto, como uma maneira de quantificar o impacto da IC na vida do paciente, nota-se a necessidade da avaliação da QV, QS e $\mathrm{SDE}$, visando um melhor direcionamento terapêutico, como também identificar os pacientes em risco. Em adição, existe interesse crescente em mensurar os fatores que afetam a QV desses pacientes, na tentativa de se obter dados que resultem em melhor intervenção clínica.
Portanto, o objetivo do estudo é relacionar a QV e a QS de pacientes com a IC comparado a indivíduos que se auto relatam saudáveis, além de correlacionar a QV com a distância percorrida no teste de caminhada de 6 minutos (TC6M) apenas nos pacientes com IC.

\section{Materiais e métodos}

\section{Sujeitos e critérios de inclusão}

Trata-se de um estudo observacional, do tipo transversal, realizado entre os anos de 2012 e 2014. Esse estudo foi aprovado pelo Comitê de Ética e Pesquisa sob o número 0147.0.051.00011. Foram incluídos 42 indivíduos, sendo 21 do grupo IC e 21 do grupo controle (GC). O grupo IC foi composto por pacientes com diagnóstico clínico de IC de diferentes etiologias, em acompanhamento ambulatorial; com quadro de IC crônica compensada, sem hospitalizações nos últimos três meses; faixa etária entre 40 e 70 anos; com classe funcional entre II a IV segundo a New York Heart Association-NYHA, categorizados pelo cardiologista que os assistiam. O GC foi composto por indivíduos auto relatados saudáveis; com idade entre 40 e 70 anos; livres de quaisquer patologias respiratórias, cardiovasculares, neuromusculares ou ortopédicas; não fumantes e/ou etilista.

Foram excluídos indivíduos com presença de déficit cognitivo, limitações ortopédicas, neuromusculares e/ou quaisquer comorbidades que interferissem em alguma etapa do protocolo, além de desistências.

\section{Dinômica do estudo}

Antes da admissão na pesquisa, os indivíduos foram informados sobre os objetivos, procedimentos e caráter metodológico do estudo, e em seguida assinaram um termo de consentimento livre e esclarecido (TCLE). Uma vez atendido aos critérios de inclusão, os indivíduos foram avaliados quanto à sua QS, SDE e QV por meio dos questionários de Pittsburg, Escala de Sonolência de 
Epworth (ESE) e 36-item Short-Form Health Survey (SF-36), respectivamente. Em seguida, foi avaliado, apenas no grupo IC, a capacidade funcional através do teste de caminhada de 6 minutos (TC6M) e a QV através do Minnesota Living with Heart Failure Questionnarie (MLHFQ). Todos os questionários foram realizados sob a forma de entrevista, aplicados pelo mesmo pesquisador, para inclusão daqueles pacientes não alfabetizados.

\section{Qualidade do sono}

A avaliação da QS foi realizada pelo Questionário de Pittsburgh, que possui dez questões abertas e semiabertas que formam sete componentes: 1) qualidade subjetiva do sono; 2) latência do sono; 3) duração do sono; 4) eficiência habitual do sono; 5) distúrbios do sono; 6) uso de medicação para dormir; 7) sonolência diurna e distúrbios durante o dia. Cada pontuação possui valores específicos, sendo 21 pontos a pontuação máxima. As pontuações superior a cinco indicam QS ruim ${ }^{13}$.

\section{Sonolência diurna excessiva}

Para avaliar a SDE foi utilizado a ESE, que avalia a probabilidade de adormecer em oito situações, envolvendo atividades diárias, que possuem um risco elevado de causar sono. As questões estão relacionadas com os hábitos de vida diários. Mesmo que o paciente nunca tenha feito algum dos itens recentemente, será estimulado a tentar descobrir como o afetariam. A cada situação serão dadas as notas mais adequadas, sendo elas: $0=$ nenhuma chance de cochilar; 1 = pequena chance de cochilar; 2 = moderada chance de cochilar e $3=$ alta chance de cochilar. A pontuação global varia de 0 a 24 , e pontuações acima de 10 sugerem presença da $\mathrm{SDE}^{14}$.

\section{Qualidade de vida}

Para avaliar a QV foram utilizados o SF36 e o MLHFQ. O SF-36 é um questionário genérico composto por 36 questões, que abordam oito domínios (ou dimensões) em dois grandes componentes: o componente físico que envolve a capacidade funcional (CF), a dor, o estado geral de saúde (ES) e limitação por aspectos físicos (AF); e o componente mental que contempla a saúde mental (SM), limitação por aspectos emocionais (AE), o aspecto social (AS) e a vitalidade (VT). Cada domínio apresenta uma pontuação final de zero a 100, no qual zero corresponde ao pior estado geral de saúde e 100 ao melhor estado de saúde ${ }^{15}$. O MLHFQ é um questionário específico para IC constituído por 21 questões objetivas, as quais avaliam as condições física, socioeconômica e as limitações emocionais. Oito questões apresentam uma forte relação com os sintomas de dispneia e fadiga e são referidas como medidas de dimensão física. Cinco outras questões estão fortemente relacionadas a questões emocionais e são referidas como medidas de dimensão emocional, e as demais questões correspondem à dimensão socioeconômica. Para cada questão, o paciente seleciona um número de 0 a 5 . Zero indica que a IC não exerce limitação e 5 indica limitação muito grande. A pontuação final do MLHFQ varia de 0 a 105 . Ao contrário do SF-36, escore elevado do MLHFQ indica pior $\mathrm{QV}^{16}$.

\section{Capacidade Funcional}

Para avaliação da capacidade funcional do grupo IC foi realizado o TC6M, que consiste na avaliação da distância máxima percorrida durante seis minutos em um corredor de 30 metros de extensão. Foram realizados dois testes idênticos com intervalo mínimo de 30 minutos, mediante recomendação da American Thoracic Society - ATS ${ }^{17}$. Os pacientes foram orientados a caminhar de acordo com sua tolerância ao exercício no período de seis minutos. Frases de incentivo foram proferidas durante a caminhada. Antes do início e ao final de cada teste, foi obtida a frequência respiratória, a frequência cardíaca e a pressão arterial, bem como a percepção de esforço por meio da Escala de Borg. O resultado 
final do TC6M foi a medida da distância total percorrida em metros durante seis minutos

\section{Anólise estatística}

Os dados foram analisados com o software SPSS versão 20.0 (SPSS Inc., Chicago, IL, USA). A análise descritiva foi apresentada em média e desvio padrão (DP). Após a evidência da distribuição normal dos dados, por meio do teste de Kolmogorov-Smirnov, utilizou-se o teste de correlação de Pearson para verificar correlação entre os domínios do SF-36 (AE e CF) com a distância percorrida do TC6M. Utilizou-se ainda o teste $t$ de student para comparações das médias dos dados antropométricos (Idade, peso, altura e IMC), dos questionários de QV, QS e ESE entre ambos os grupos, grupo IC e GC. O nível de significância para os testes utilizados foi de $5 \%$.

\section{Resultados}

Foram recrutados 73 (idade: $55 \pm 8,36$ anos) indivíduos. Dos 52 pacientes com IC, 31 foram excluídos por causa da inviabilidade de condução ao local de avaliação, da impossibilidade de compreender os questionários ou da incompatibilidade com compromissos familiares que impediam a visita ao ambulatório onde seria realizada a pesquisa. Ao final, apenas 21 pacientes com IC e 21 indivíduos saudáveis concluíram o estudo.

As características clínicas e antropométricas da amostra encontram-se na Tabela 1. O grupo IC apresentou alta incidência do uso de fármacos da classe de betabloqueadores $(90 \%-$ 19 sujeitos). Ainda no grupo IC, 71,4\% (15) faziam uso de diuréticos; $38,1 \%$ (8) faziam uso de antiagregantes; $23,8 \%$ (5) faziam uso de hipolipemiantes; e 19\% (4) faziam uso de antiarrítmicos. Cerca de $80 \%$ (17) dos pacientes usavam marca-passo.

Ao avaliar a QS pelo Questionário de Pittsburgh, foi observado que $52,4 \%$ do grupo IC apresentou QS ruim (score $>5$ ) comparado a apenas 4,8\% do GC. Quanto à sonolência diurna,
Tabela l: Características gerais da população estudada

\begin{tabular}{|c|c|c|c|}
\hline & Grupo IC & $\begin{array}{c}\text { Grupo } \\
\text { controle }\end{array}$ & P-valor \\
\hline Idade (anos) & $55 \pm 8,36$ & $54,84 \pm 7,91$ & 0,95 \\
\hline \multicolumn{4}{|l|}{ Sexo } \\
\hline $\begin{array}{l}\text { Feminino } \\
\text { Masculino }\end{array}$ & $\begin{array}{c}8(38,1 \%) \\
13(61,9 \%) \\
\end{array}$ & $\begin{array}{l}8(38,1 \%) \\
13(61,9 \%)\end{array}$ & \\
\hline$P_{(\mathrm{kg})}$ & $70 \pm 10$ & $68 \pm 11$ & 0,85 \\
\hline Altura $_{(\mathrm{m})}$ & $162,71 \pm 5,54$ & $163,10 \pm 8,72$ & 0,86 \\
\hline$I M C_{(\mathrm{kg} / \mathrm{m})^{2}}$ & $26,35 \pm 3,75$ & $25,82 \pm 2,60$ & 0,59 \\
\hline \multicolumn{4}{|c|}{ Classe Funcional (NYHA) } \\
\hline $\begin{array}{l}\text { II } \\
\text { III }\end{array}$ & $\begin{array}{l}12(57,15 \%) \\
9(42,85 \%)\end{array}$ & - & - \\
\hline FEVE (\%) & $33 \pm 10,61$ & - & - \\
\hline MLHFQ & $27 \pm 17$ & - & - \\
\hline \multicolumn{4}{|c|}{$\begin{array}{l}\text { Dados apresentados em média } \pm \text { desvio padrão. } \\
\text { IMC: índice de massa corpórea; NYHA: New York } \\
\text { Heart Association; FEVE: fração de ejeção ventricular } \\
\text { esquerda. p } \leq 0,05 \text { para significância estatística. } \\
\text { Fonte: Os autores. }\end{array}$} \\
\hline
\end{tabular}

$42,8 \%$ do grupo IC apresentou pontuações indicativas de SDE (escores > 10) e 52,4\% apresentou ESE > 8. No GC, nenhum indivíduo apresentou SDE e apenas 19\% apresentou ESE > 8 (Tabela 2). A comparação entre os scores do questionário de Pittsburgh e ESE está representada na figura 1.

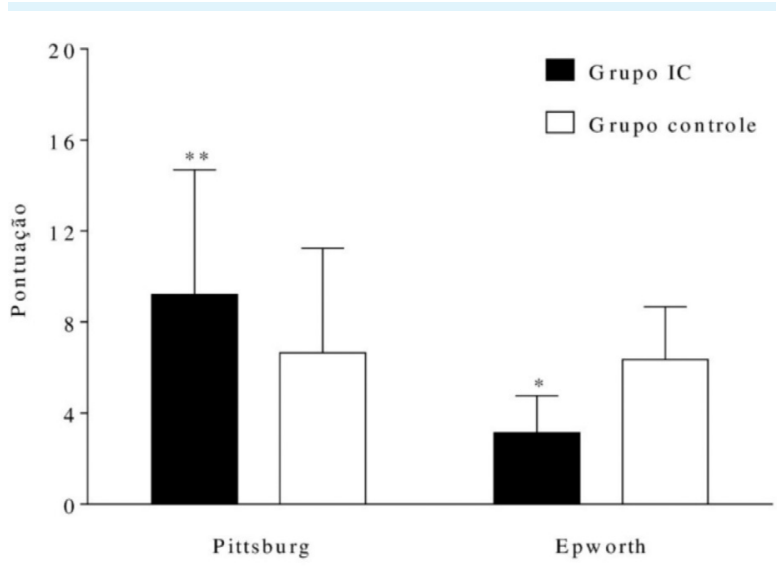

Figura 1: Comparação dos scores do questionório Pittsburg e escala de sonolência de Epworth entre o grupo IC e o grupo controle Dados apresentados em média \pm desvio padrão .*, ${ }^{* *}: \mathrm{p}<0.05, \mathrm{p}<0.005$, teste $t$ de student Fonte: Os autores. 
Ao avaliar a QV, o grupo IC apresentou uma pontuação menor ( $\mathrm{p}<0,005)$ nos domínios $\mathrm{CF}, \mathrm{ES}, \mathrm{AF}, \mathrm{SM}, \mathrm{AE}, \mathrm{AS}$ e a VT, mostrado na tabela 2 .

Tabela 2: Comparação da qualidade de vida entre o grupo IC e o grupo controle

\begin{tabular}{cccc}
\hline & Grupo IC & Grupo Controle & P-valor \\
\hline CF & $53,56 \pm 25,39$ & $87,62 \pm 11,89$ & $0,000^{*}$ \\
AF & $31,25 \pm 34,29$ & $83,33 \pm 30,95$ & $0,000^{*}$ \\
DOR & $61,20 \pm 27,78$ & $74,90 \pm 17,56$ & 0,065 \\
ES & $61,95 \pm 23,00$ & $81,52 \pm 12,03$ & $0,002^{*}$ \\
VT & $59,50 \pm 20,89$ & $77,62 \pm 15,93$ & $0,003^{*}$ \\
AS & $68,75 \pm 21,65$ & $92,95 \pm 9,27$ & $0,000^{*}$ \\
AE & $60,78 \pm 39,50$ & $90,46 \pm 26,16$ & $0,008^{*}$ \\
SM & $69,40 \pm 22,60$ & $85,81 \pm 14,33$ & $0,009^{*}$ \\
\hline
\end{tabular}

Dados apresentados em média \pm desvio padrão. CF: capacidade funcional; LF: limitação por aspectos físicos; ES: estado geral de saúde; VT: vitalidade; AS: Aspectos sociais; AE: limitação por aspectos emocionais; SM: Saúde Mental. ${ }^{*} p \leq 0,05$ para significância estatística.

Fonte: Os autores.

Além disso, ao correlacionar a $\mathrm{QV}$, medida pelo SF-36, com a distância percorrida do TCM6, foi observado uma correlação forte no domínio $\mathrm{AE}(\mathrm{r}=0,62, \mathrm{p}=0,004)$, e moderada no domínio $\mathrm{CF}$ $(\mathrm{r}=0,47, \mathrm{p}=0,03)$.

\section{Discussão}

Devido a importância de avaliar o impacto que a IC causa na QV e QS, o nosso estudo objetivou relacionar a QV e a QS de pacientes com IC comparado a saudáveis e correlacionar a QV com a distância percorrida no TC6M apenas nos pacientes com IC. Os principais achados foram: 1) O grupo IC apresentou uma pior QV e QS comparado aos saudáveis; 2) Os domínios AE e CF apresentaram uma correlação positiva com a distância percorrida no TC6M.

Há um interesse crescente na ligação dos distúrbios respiratórios do sono (DRS) e a insuficiência cardíaca crônica (ICC) devido as semelhanças na fisiologia de ambos. Na ICC foram reconhecidos dois DRS: a apneia obstrutiva do sono (AOS) e apneia central do sono (ACS). A AOS como a ACS interferem no sistema neurohumoral e assim agravam a IC, por exemplo, pelo aumento da atividade nervosa simpática e do eixo renina-angiotensina-aldosterona, ambos alvos das terapias de última geração para $\mathrm{IC}^{8-}$ 10,18,19. Vários estudos demonstraram um aumento da mortalidade de pacientes com ICC portadores de DRS, em contraste com aqueles sem DRS 20,18,19. Estudos recentes têm mostrado a importância da realização de trabalhos de triagem sobre alterações do sono na prática clínica dos profissionais que lidam com IC ${ }^{7}$. Como esperado no nosso estudo, foi observado que o grupo IC apresentou uma maior predominância de indivíduos $(52,4 \%)$ com a pontuação do questionário de Pittsburgh superior a 5, remetendo a uma QS ruim. Nossos resultados concordam com o estudo de Azevedo et al..$^{21}$, que avaliou pacientes com IC classe funcional II e III, dos quais cerca de 60,9\% (14) da amostra apresentaram valores do Questionário de Pittsburgh maiores que 5, sendo classificados "como mal dormidores". Outro estudo realizado em Taiwan que incluiu 101 pacientes com IC (idade média $=74$ anos; classe funcional II $=63,4 \%$ ), também observou uma alta prevalência de pacientes com a QS ruim ${ }^{22}$.

A SDE também é um distúrbio do sono comumente encontrado em pacientes com $\mathrm{IC}^{7,21}$, no nosso estudo $42,8 \%$ do grupo IC obteve pontuação na ESE superior a 10. Redeker et al. ${ }^{23}$, avaliou pacientes com IC com classe funcional I-IV, e também observou uma alta prevalência $(44,1 \%)$ de pacientes com SDE. A elevada quantidade de pacientes com IC com SDE, é uma consequência específica do sono perturbado ${ }^{23}$. Foi observado que indivíduos com sono deficiente, devido a limitações na qualidade ou quantidade do sono, são incapazes de responder rapidamente a estímulos externos e possuem uma maior dificuldade de concentração, prejudicando sua capacidade de realizar algumas atividades e sua qualidade de vida ${ }^{24}$.

A QV vem assumindo importância, sob vários aspectos nos últimos anos como uma fer- 
ramenta que demonstra a eficácia de várias intervenções terapêuticas na IC ${ }^{25}$. No que se refere a avaliação da QV feita através do SF-36, observamos que os resultados alcançados evidenciam pontuações significantemente mais baixas no grupo IC em 7 domínios (CF, AF, ES, VT, AS, AE e SM) quando comparado ao GC. O estudo realizado por Johansson et al. ${ }^{26}$, avaliou 109 pacientes com IC, classe funcional I a III, cuja média de idade foi de 79,5 anos para o grupo com IC e de 78 anos para o grupo sem doença cardiovascular, e também evidenciou diferenças significativas em domínios semelhantes, corroborando com os nossos resultados. Estudos anteriores, confirmam que sujeitos com IC apresenta baixa qualidade de vida quando comparado com sujeitos saudáveis ${ }^{23}$. Possivelmente devido a população estudada apresentar dispneia e fadiga precoce, característica da patologia, que implica diretamente na QV desses pacientes ${ }^{26}$.

Ao relacionar os questionários de $\mathrm{QV}$, o específico (MLHFQ) e o genérico (SF-36) no grupo IC encontramos baixas correlações. Isso pode ser explicado, pois MLHFQ foi desenvolvido especificamente para IC, o que o torna mais próximo à realidade desse tipo de paciente, avaliando sua condição de enfermidade. Por sua vez, o questionário genérico SF-36 foi desenvolvido para avaliar a condição de saúde em pacientes com doença crônica, o que pode ter determinado a diferença encontrada no estudo ${ }^{15,27}$.

Avaliamos o impacto da QV na capacidade funcional dos pacientes com IC através da correlação dos domínios do questionário SF-36 e a distância percorrida do TC6M. Observamos uma correlação forte no domínio AE e moderada no domínio CF do grupo IC. O estudo de Nogueira et al. ${ }^{27}$. também observou uma correlação entre o domínio AE e a distância percorrida no TC6M nos pacientes com IC. A dispneia e a fadiga apresentadas pelos pacientes com IC interferem nas atividades de vida diária dessa população. Esses sintomas atuam diretamente de forma a causar prejuízos tanto na QV como na capacidade funcional desses pacientes, sendo de fundamental importância a mensuração, através de questionários e testes funcionais por parte dos profissionais da saúde, dos prejuízos causados pela doença.

\section{Limitações do estudo}

Nosso estudo apresentou algumas limitações, tais como o tamanho reduzido da amostra e a subjetividade dos indivíduos auto relatados saudáveis. Sugere-se a divisão por classe funcional, pois nossos resultados não se aplicam as demais classificações.

\section{Conclusão}

Nossos resultados demonstram que a IC gera impactos negativos na QV e na QS da amostra estudada, como também a presença de SDE. Acreditamos que nosso estudo possui relevância clínica pois mostra a importância da avaliação e do tratamento multiprofissional, por parte dos profissionais da saúde, dos prejuízos na saúde que a IC causa nos pacientes com essa doença.

\section{Referências}

1 Real J, Cowles E, Wierzbicki AS, Guideline Committee. Chronic heart failure in adults: summary of updated NICE guidance. BMJ 2018; 362: k3646.

2 De Maeyer C, Beckers P, Vrints CJ, Conraads VM. Exercise training in chronic heart failure. Ther Adv Chronic Dis 2013; 4: 105-17.

3 Daher A, Matthes M, Keszei A, Brandenburg V, Müller T, Cornelissen C et al. Characterization and Triggers of Dyspnea in Patients with Chronic Obstructive Pulmonary Disease or Chronic Heart Failure: Effects of Weather and Environment. Lung 2018. doi:10.1007/s00408-018-0170-2.

4 Herr JK, Salyer J, Lyon DE, Goodloe L, Schubert C, Clement DG. Heart Failure Symptom Relationships. J Cardiovasc Nurs 2014; 29: 416-422.

5 Yancy CW, Jessup M, Bozkurt B, Butler J, Casey DE, Drazner MH et al. 2013 ACCF/AHA Guideline for the Management of Heart Failure: Executive Summary. Circulation 2013; 128: 1810-1852. 
6 Giallauria F, Piccioli L, Vitale G, Sarulli FM. Exercise training in patients with Chronic Heart Failure: a new challenge for Cardiac Rehabilitation Community. Monaldi Arch Chest Dis 2018; 88: 987.

7 Oldenburg O, Lamp B, Faber L, Teschler H, Horstkotte D, Töpfer V. Sleep-disordered breathing in patients with symptomatic heart failure: a contemporary study of prevalence in and characteristics of 700 patients. Eur J Heart Fail 2007; 9: 251-7.

8 Terziyski K, Draganova A. Central Sleep Apnea with Cheyne-Stokes Breathing in Heart Failure From Research to Clinical Practice and Beyond. In: Advances in experimental medicine and biology. 2018, pp 327-351.

9 Drager LF, McEvoy RD, Barbe F, Lorenzi-Filho G, Redline S, INCOSACT Initiative (International Collaboration of Sleep Apnea Cardiovascular Trialists). Sleep Apnea and Cardiovascular Disease. Circulation 2017; 136: 1840-1850.

10 Cowie MR, Gallagher AM. Sleep Disordered Breathing and Heart Failure. JACC Hear Fail 2017; 5: 715-723.

11 Riegel B, Ratcliffe SJ, Sayers SL, Potashnik S, Buck HG, Jurkovitz $C$ et al. Determinants of excessive daytime sleepiness and fatigue in adults with heart failure. Clin Nurs Res 2012; 21: 271-93.

12 Sousa MM de, Oliveira JS, Soares MJGO, Bezerra SMMS, Araújo AA de, Oliveira SHS et al. Associação das condições sociais e clínicas à qualidade de vida de pacientes com insuficiência cardíaca. Rev Gaúcha Enferm 2017;38(2):e65885.

13 Bertolazi AN, Fagondes SC, Hoff LS, Dartora EG, da Silva Miozzo IC, de Barba MEF et al. Validation of the Brazilian Portuguese version of the Pittsburgh Sleep Quality Index. Sleep Med 2011; 12: 70-75.

14 Bertolazi NA, Fagondes SC, Hoff LS, Dallagasperina PV, Barreto SSM, Johns MW. Validação da escala de sonolência de Epworth em português para uso no Brasil. J Bras Pneumol 2009; 35: 877-883.

15 Ciconelli RM, Ferraz MB, Santos W, Meinão I, Quaresma MR. Tradução para a língua portuguesa e validação do questionário genérico de avaliação de qualidade de vida SF-36 (Brasil SF-36). Rev. Bras. Reumatol. 1999; 39: 143-150.

Riegel B, Moser DK, Glaser D, Carlson B, Deaton C, Armola R et al. The Minnesota Living With Heart Failure Questionnaire: sensitivity to differences and responsiveness to intervention intensity in a clinical population. Nurs Res 2002; 51: 209-18.
17 ATS Statement: Guidelines for the Six-Minute Walk Test. Am Thorac Soc Am J Respir Crit Care Med 2002; 166: 111-117.

18 Hanly PJ, Zuberi-Khokhar NS. Increased mortality associated with Cheyne-Stokes respiration in patients with congestive heart failure. Am J Respir Crit Care Med 1996; 153: 272-276.

19 Lanfranchi PA, Braghiroli A, Bosimini E, Mazzuero G, Colombo R, Donner CF et al. Prognostic value of nocturnal Cheyne-Stokes respiration in chronic heart failure. Circulation 1999; 99: 1435-40.

20 Milleron O, Pillière R, Foucher A, de Roquefeuil F, Aegerter P, Jondeau G et al. Benefits of obstructive sleep apnoea treatment in coronary artery disease: a long-term follow-up study. Eur Heart J 2004; 25: 728-734.

21 Azevedo IG, Vieira EM de A, Oliveira Neto NR de, Nogueira IDB, Melo FES de, Nogueira PA de MS et al. Correlation between sleep and quality of life in patients with heart failure. Fisioter e Pesqui 2015; 22: 148-154.

22 Wang T-J, Lee S-C, Tsay S-L, Tung H-H. Factors influencing heart failure patients' sleep quality. J Adv Nurs 2010; 66: 1730-1740.

23 Redeker NS SS. Characteristics of sleep in patients with stable heart failure versus a comparison group. Hear Lung 2006; 35: 252-61.

24 Antonelli Incalzi R, Marra C, Salvigni BL, Petrone A, Gemma A, Selvaggio D et al. Does cognitive dysfunction conform to a distinctive pattern in obstructive sleep apnea syndrome? J Sleep Res 2004; 13: 79-86.

25 Saccomann ICR, Cintra FA, Gallani MCBJ. Qualidade de vida relacionada à Saúde em Idosos com Insuficiência cardíaca: avaliação com instrumento específico. Acta Paul Enferm 2011; 24: 179-184.

26 Johansson P, Arestedt K, Alehagen U, Svanborg E, Dahlström U, Broström A. Sleep disordered breathing, insomnia, and health related quality of life -- a comparison between age and gender matched elderly with heart failure or without cardiovascular disease. Eur J Cardiovasc Nurs 2010; 9: 108-17.

27 Nogueira IDB, Servantes DM, Nogueira PA de MS, Pelcerman A, Salvetti XM, Salles F et al. Correlação entre qualidade de vida e capacidade funcional na insuficiência cardíaca. Arq Bras Cardiol 2010; 95: 238-243. 